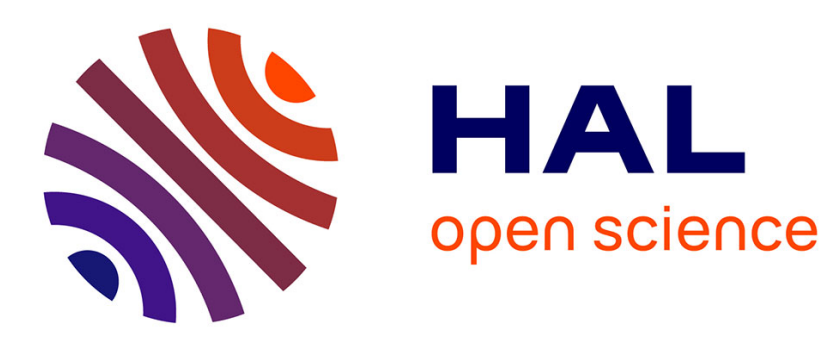

\title{
Conductivité thermique du béton cellulaire autoclavé: modélisation par méthode autocohérente
}

\author{
C. Boutin
}

\section{To cite this version:}

C. Boutin. Conductivité thermique du béton cellulaire autoclavé: modélisation par méthode autocohérente. Materials and structures, 1996, 29 (10), pp.609-615. hal-00941106

\author{
HAL Id: hal-00941106 \\ https://hal.science/hal-00941106
}

Submitted on 3 Feb 2014

HAL is a multi-disciplinary open access archive for the deposit and dissemination of scientific research documents, whether they are published or not. The documents may come from teaching and research institutions in France or abroad, or from public or private research centers.
L'archive ouverte pluridisciplinaire HAL, est destinée au dépôt et à la diffusion de documents scientifiques de niveau recherche, publiés ou non, émanant des établissements d'enseignement et de recherche français ou étrangers, des laboratoires publics ou privés. 


\title{
Conductivité thermique du béton cellulaire autoclavé: modélisation par méthode autocohérente
}

\author{
(Thermal conductivity of autoclaved aerated concrete: modelling by the self-consistent method)
}

\author{
C. Boutin \\ École Nationale des Travaux Publics de l'État, Laboratoire Géomatériaux, DGCB, URA CNRS N¹652, Rue Maurice Audin, \\ 69518 Vaulx-en-Velin Cedex, France
}

\section{R É S U M É}

Dans cet article, nous utilisons la méthode autocohérente pour déterminer la conductivité thermique du béton cellulaire autoclavé selon sa densité et sa teneur en eau. On montre que cette méthode est bien adaptée au béton cellulaire du fait de la présence de pores de tailles très différentes. Nous faisons l'hypothèse que la conductivité thermique et la densité des particules solides sont identiques quelque soit la densité du béton cellulaire. À partir de données expérimentales ces propriétés sont estimées.

Pour traiter le béton cellulaire $\mathrm{sec}$, on utilise directement les résultats classiques de la méthode autocohérente à inclusions composites, obtenus en considérant que le matériau est constitué de bulles d'air entourées d'une membrane solide. Compte tenu des valeurs de conductivité de l'air et des particules solides, et des densités habituelles, les expressions théoriques peuvent être simplifiées. La conductivité à l'état sec ainsi obtenue est en excellent accord avec les données expérimentales.

Pour le béton cellulaire humide, deux modélisations sont examinées. La première est une modélisation autocohérente avec des inclusions triphasées, où une couche d'eau est intercalée entre l'air et la membrane solide. Dans cette description, la concentration de l'eau est la même quelle que soit la taille des pores. Pour les valeurs usuelles de densité et de teneur en eau, le résultat n'est qu'en accord qualitatif avec les expériences. Ceci peut être attribué à une mauvaise description de la répartition de l'eau dans les pores.

Dans la seconde approche, nous procédons par "double homogénéisation". Le matériau est modélisé en considérant des bulles d'air entourées d'une membrane de milieu poreux saturé. La conductivité ainsi obtenue est en bon accord avec les données expérimentales.

\section{A B S T T R A C T}

The self-consistent method was used to determine the thermal conductivity of autoclaved aerated concrete according to its dry density and moisture. We show that this method is efficient for autoclaved aerated concrete as its microstructure contains very different-sized pores. The basic hypothesis is that the density and the thermal conductivity of the solid particles are identical regardless of the dry density of the autoclaved aerated concrete. These properties were assessed from experimental data.

When dealing with dry autoclaved aerated concrete, we use the results of the self-consistent method applied to composite inclusions. We assume that dry autoclaved aerated concrete is constituted of air bubbles of various sizes, embedded in a solid membrane. Taking into account the values of conductivity of air and solid particles, and the typical range of density, the theoretical expressions can be simplified. The dry conductivity thus obtained is in excellent agreement with the experimental data.

When dealing with wet autoclaved aerated concrete, two models were proposed. The first is an extension of the self-consistent approach applied to the case of three-phase composite inclusions: a water layer is located between the air and the solid membrane. In this description, the water concentration is the same whatever the pore size. Within the normal range of variation of moisture and density, this result agrees qualitatively, but not quantitatively, with the experiments. This is probably due to the rough description of water distribution.

In the second approach, a "double homogenization" procedure is applied. In this way, the description of the wet autoclaved aerated concrete microstructure is improved since $d r y$ air bubbles are embedded in a saturated porous media. Then, wet thermal conductivity is in good agreement with the experiments. 


\section{NOTATIONS ET ORDRES DE GRANDEUR DES PARAMĖTRES}

$\mathrm{R}_{\mathrm{a}}, \mathrm{R}_{\mathrm{s}}, \mathrm{R}_{\mathrm{w}}$ : Rayons des sphères utilisées pour décrire un matériau hétérogène par la méthode autocohérente à inclusions composites

$\mathfrak{u}_{\mathrm{m}}$ : Teneur en eau massique du béton cellulaire humide (de 0 à 0,40$)$

$\delta: \quad$ Concentration volumique de l'eau dans les pores (de 0 à 0,5$)$

$\varepsilon$ : $\quad$ Porosité d'ensemble du béton cellulaire (de 0,65 à 0,85 )

$\varepsilon_{\mathrm{W}}$ : $\quad$ Concentration volumique de l'eau dans les pores saturés

$\varepsilon_{\mathfrak{a}}$ : Concentration d'air dans le béton cellulaire humide

$\lambda$ : $\quad$ Conductivité thermique (W/mK)

$\lambda_{\mathrm{a}}$ : $\quad$ Conductivité thermique de l'air $(0,026$ $\mathrm{W} / \mathrm{mK}$ )

$\lambda_{\mathrm{d}}$ : $\quad$ Conductivité thermique du béton cellulaire sec (de 0,08 à $0,2 \mathrm{~W} / \mathrm{mK}$ )

$\lambda_{\mathrm{s}}$ : $\quad$ Conductivité thermique des particules solides $(\approx 0,89 \mathrm{~W} / \mathrm{mK})$

$\lambda_{\mathrm{u}}$ : $\quad$ Conductivité thermique du béton cellulaire humide (de 0,08 à $0,3 \mathrm{~W} / \mathrm{mK}$ )

$\lambda_{\mathrm{W}}$ : $\quad$ Conductivité thermique de l'eau $(0,602$ $\mathrm{W} / \mathrm{mK}$ )

$\lambda_{\text {sw }}$ : Conductivité thermique de la partie saturée du béton cellulaire humide

$\rho_{\mathrm{d}}$ : $\quad$ Masse volumique du béton cellulaire sec (de 350 à $850 \mathrm{~kg} / \mathrm{m}^{3}$ )

$\rho_{\mathrm{s}}$ : $\quad$ Masse volumique des particules solides $\left(\approx 2650 \mathrm{~kg} / \mathrm{m}^{3}\right)$

$\rho_{\mathrm{w}}$ : $\quad$ Masse volumique de l'eau $\left(10^{3} \mathrm{~kg} / \mathrm{m}^{3}\right)$

\section{INTRODUCTION}

Le béton cellulaire autoclavé (AAC) est un matériau de construction dont les deux principaux avantages sont d'une part sa légèreté, d'autre part sa faible conductivité thermique.

Pour répondre aux exigences des constructeurs sur les qualités thermiques et mécaniques du béton cellulaire autoclavé, les fabricants proposent une gamme de matériaux dont la densité varie de $350 \mathrm{~kg} / \mathrm{m}^{3}$ à $800 \mathrm{~kg} / \mathrm{m}^{3}$. Ce paramètre influence fortement la résistance mécanique, qui est d'autant plus forte que la densité est élevée, et les propriétés d'isolation, qui sont meilleures à faible densité. Par ailleurs, on sait que pour une même densité, la conductivité thermique augmente significativement avec la teneur en eau du matériau.

Comme le pouvoir d'isolation est une qualité essentielle du AAC, des études expérimentales ont été réalisées en laboratoire sur des murs ou des plaques [1-5]. Les mesures étant à la fois longues, coûteuses et délicates, certains auteurs [6] ont proposé une modélisation théorique des propriétés thermiques. Cependant il n'existe pas de résultats théoriques permettant d'exprimer simplement la conductivité thermique à partir de la densité et de la teneur en eau du AAC. Les seules relations disponibles actuellement sont obtenues par des régressions linéaires sur des résultats expérimentaux.

Dans cet article, on se propose de déterminer les propriétés thermiques du béton cellulaire sec ou humide en appliquant la méthode autocohérente. Cette approche, qui a été développée initialement pour l'élasticité et l'élastoplasticité par [7], a aussi été appliquée aux problèmes de conductivité [8]. La méthode permet d'estimer les caractéristiques d'ensemble de matériaux hétérogènes, en s'appuyant sur la description physique des phénomènes ayant lieu dans une cellule représentative du matériau.

Pour le béton cellulaire autoclavé sec, la cellule représentative est constituée d'une bulle de gaz entourée d'une membrane solide dont les rayons relatifs dépendent de la densité. Dans le domaine de variation usuel de la densité, on déduit des expressions très générales obtenues, des relations simples dont on montre le très bon accord avec les observations expérimentales déjà publiées, en particulier par $[4,5]$.

Pour le béton cellulaire humide, deux modélisations sont examinées. La plus simple consiste à modifier le modèle précédent en intercalant entre l'air et la membrane solide une couche d'eau dont l'épaisseur dépend de la teneur en eau. Les résultats sont en accord qualitatif avec l'expérience mais sous-estiment l'effet de la teneur en eau. Pour améliorer la description, nous présentons ensuite une modélisation plus représentative du béton cellulaire humide, où des bulles d'air sont entourées d'une membrane constituée du matériau poreux saturé, dans des proportions liées à la teneur en eau. Les propriétés thermiques déterminées par cette approche théorique sont cohérentes avec les mesures expérimentales.

Dans la deuxième partie de cet article, on présente succinctement la méthode et son applicabilité au AAC du fait de la présence de pores de tailles très différentes. Dans la troisième partie et la quatrième partie, on applique la méthode au AAC sec, puis au AAC humide, et dans les deux cas les expressions théoriques sont comparées aux relations empiriques.

\section{LA MÉTHODE AUTOCOHÉRENTE}

L'objectif de la méthode autocohérente, dans le domaine thermique, est d'estimer la conductivité d'ensemble d'un matériau hétérogène à partir de la concentration des constituants et de leur conductivité propre. L'idée essentielle est d'assimiler l'interaction de chaque hétérogénéité avec toutes les autres, à l'interaction de cette hétérogénéité avec une matrice homogène dont les propriétés sont à déterminer. Le principe de la détermination est fondé sur une condition énergétique qui exprime que l'énergie thermique contenue dans le matériau hétérogène est égale à celle qu'il y aurait dans la matrice homogène équivalente soumise aux même conditions aux limites.

L'avantage de cette approche est de ramener le problème initial à la résolution de problèmes élémentaires 
d'interaction entre matrice homogène et hétérogénéités. De ce fait, pour des géométries d'inclusions suffisamment simples, il est possible d'exprimer la conductivité d'ensemble sous forme analytique.

\subsection{Conductivité d'un milieu à deux phases}

Dans ce paragraphe, nous considérerons un milieu constitué "d'inclusions composites sphériques". Il s'agit d'une géométrie où une sphère du constituant «a» de conductivité $\lambda_{a}$ et de rayon $R_{a}$ est entourée par une coquille concentrique du constituant "s" de conductivité $\lambda_{s}$ et de rayon $R_{s}$ (voir Fig. 1). Cette configuration permet d'assurer la connexité de la phase externe, indépendamment de la concentration volumique de la phase interne $\varepsilon$.

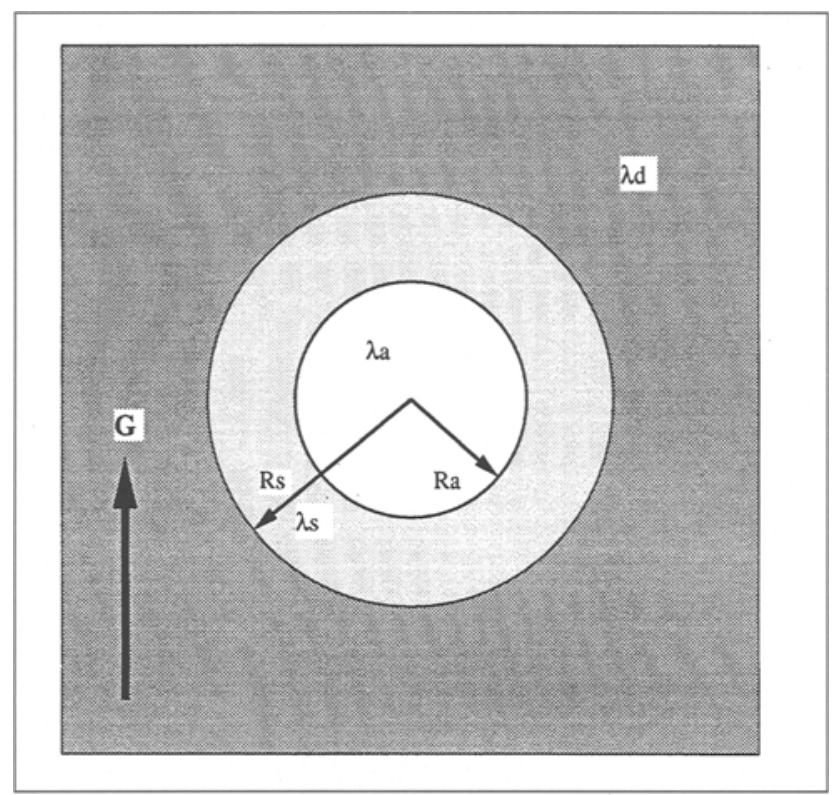

Fig. 1 - Géométrie d'une inclusion composite élémentaire prise en compte dans la méthode autocohérente appliquée aux milieux à deux phases.

Rappelons brièvement la procédure de calcul de la conductivité équivalente [8].

Déterminons dans un premier temps le champ de température se développant dans une sphère composite noyée dans un milieu de conductivité $\lambda_{\mathrm{e}}$ et subissant à l'infini un gradient de température uniforme unitaire $\mathrm{G}$.

Pour chaque milieu $(i=a, s, e)$, la température et le flux de chaleur doivent être continus aux frontières, et vérifier l'équation de la chaleur:

$$
\operatorname{div}\left(\lambda_{i} \operatorname{grad} T_{i}\right)=\lambda_{i} \Delta T_{i}=0
$$

Le problème est à symétrie de révolution et, en coordonnées sphériques (l'axe $\theta=0$ étant orienté selon $G$ ), le champ de température ne dépend que des variables $r$ et $\theta$. Compte tenu de la géométrie, on montre simplement que la solution de (1) est du type:

$$
T_{i}=\left(A_{i} \cdot r+B_{j} / r^{2}\right) \cos \theta \quad i=A, s, e
$$

Les constantes sont déterminées par les conditions aux limites.
En $\mathrm{r}=0$ la température $\mathrm{T}_{\mathrm{a}}$ est finie d'où : $\mathrm{B}_{\mathrm{a}}=0$

À l'infmi le gradient tend vers $G$ d'où : $A_{e}=1 \quad(|G|=1)$

À la frontière des milieu «a», $\left(r=R_{a}\right)$ et « $s$ », $\left(r=R_{s}\right)$ on a :

- la continuité de température, soit :

$$
\begin{array}{ll}
A_{a} R_{a}=A_{s} \cdot R_{a}+B_{s} / R_{a}^{2} & \left(\text { en } r=R_{a}\right) \\
A_{s} R_{s}+B_{s} / R_{s}^{2}=R_{s}+B_{e} / R_{s}^{2} & \left(\text { en } r=R_{s}\right)
\end{array}
$$

- la continuité de flux:

$$
\begin{array}{ll}
\left(\lambda_{\mathrm{a}} \operatorname{grad} \mathrm{T}_{\mathrm{a}}\right) \cdot \mathrm{e}_{\mathrm{r}}=\left(\lambda_{\mathrm{s}} \operatorname{grad} \mathrm{T}_{\mathrm{s}}\right) \cdot \mathrm{e}_{\mathrm{r}} & \left(\mathrm{en} \mathrm{r}=\mathrm{R}_{\mathrm{a}}\right) \\
\left(\lambda_{\mathrm{s}} \operatorname{grad} \mathrm{T}_{\mathrm{s}}\right) \cdot \mathrm{er}=\left(\lambda_{\mathrm{e}} \operatorname{grad} \mathrm{T}_{\mathrm{e}}\right) \cdot \mathrm{e}_{\mathrm{r}} & \left(\text { en } \mathrm{r}=\mathrm{R}_{\mathrm{e}}\right)
\end{array}
$$

soit :

$$
\begin{aligned}
& \lambda_{a} A_{a}=\lambda_{s} \cdot\left(A_{s}-2 B_{s} / R_{a}{ }^{3}\right) \\
& \lambda_{s} \cdot\left(A_{s}-2 B_{s} / R_{s}{ }^{3}\right)=\lambda_{e} \cdot\left(1-2 B_{e} / R_{s}{ }^{3}\right)
\end{aligned}
$$

Introduisons maintenant l'hypothèse de la méthode autocohérente. La conductivité $\lambda_{\mathrm{e}}$ doit être telle que, sous le même gradient à l'infini $G$, il y ait identité entre les énergies thermiques contenues:

- dans le milieu homogène équivalent $\left(\lambda_{\mathrm{e}}\right)$ sans inclusions,

- dans le milieu $\left(\lambda_{\mathrm{e}}\right)$ avec l'inclusion composite $\left(\lambda_{\mathrm{a}}, \lambda_{\mathrm{s}}\right)$.

Une condition équivalente est que la moyenne du gradient de température dans la sphère composite est égale au gradient $\mathrm{G}$, [9].

Après calcul, cette condition se ramène à $B_{e}=0$. Ainsi, les équations (2) et (3) constituent un système de 4 équations à 3 inconnues, qui n'a de solution que si son déterminant est nul, ce qui conduit à l'expression de la conductivité d'ensemble:

$$
\lambda_{\mathrm{e}}=\lambda_{\mathrm{s}}\left[1+\frac{\varepsilon}{\frac{1-\varepsilon}{3}+\frac{1}{\lambda_{\mathrm{a}} / \lambda_{\mathrm{s}}-1}}\right]
$$

Dans cette expression théorique (4) il est supposé que la concentration de la phase non connexe est égale au rapport des volumes des sphères internes et externes:

$$
\varepsilon=\left(\mathrm{R}_{\mathrm{a}} / \mathrm{R}_{\mathrm{s}}\right)^{3}
$$

Comme cela à été démontré en particulier dans [10], cette hypothèse n'est satisfaite que si le matériau est constitué d'un assemblage de sphères composites de tailles variables (voir Fig. 2).

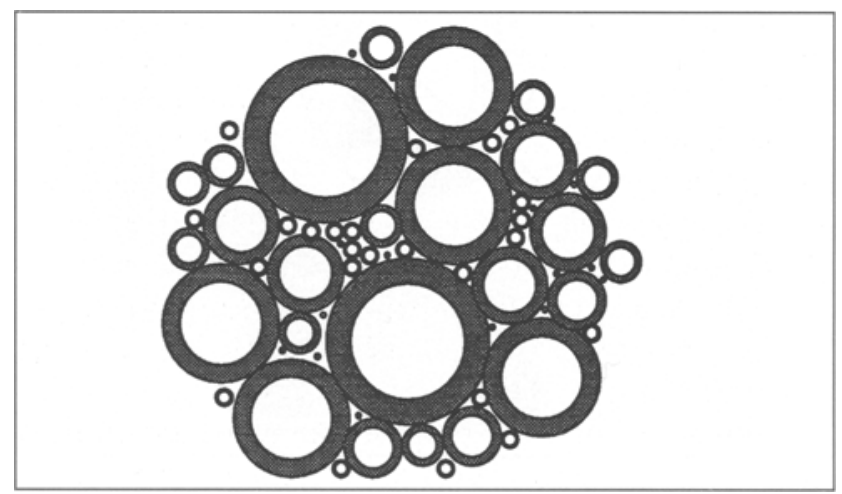

Fig. 2 - Structure d'un matériau hétérogène correspondant aux hypothèses de la méthode autocohérente. 


\subsection{Conductivité d'un milieu à trois phases}

La méthode permettant de traiter les matériaux formés d'inclusions composites sphériques à deux constituants, peut facilement être étendue aux milieux à trois phases. La géométrie considérée correspond alors à une sphère (de rayon $R_{a}$ et de conductivité $\lambda_{a}$ ) entourée par une coquille concentrique (d'épaisseur $R_{w}-R_{a}$ et conductivité $\lambda_{w}$ ) entourée elle-même par une coquille concentrique du constituant de conductivité $\lambda_{s}$ d'épaisseur $\mathrm{R}_{\mathrm{s}}-\mathrm{R}_{\mathrm{W}}$ (voir Fig. 3). En admettant toujours que les concentrations de phases sont données par les rapports des volumes des différentes sphères, et en notant :

$$
\varepsilon=\left(\mathrm{R}_{\mathrm{w}} / \mathrm{R}_{\mathrm{s}}\right)^{3} \quad \delta=1-\left(\mathrm{R}_{\mathrm{a}} / \mathrm{R}_{\mathrm{w}}\right)^{3}
$$

La conductivité d'ensemble est alors donnée par l'expression (5):

$$
\lambda_{\mathrm{e}}=\lambda_{\mathrm{s}}\left[1+\frac{\varepsilon}{\frac{1-\varepsilon}{3}+\frac{1+\delta\left(\lambda_{\mathrm{a}} / \lambda_{\mathrm{w}}-1\right) / 3}{\lambda_{\mathrm{a}} / \lambda_{\mathrm{s}}-1-\delta\left(\lambda_{\mathrm{a}} / \lambda_{\mathrm{w}}-1\right)\left(2 \lambda_{\mathrm{w}} / \lambda_{\mathrm{s}}+1\right) / 3}}\right]
$$

\subsection{Microstructure du béton cellulaire}

Les études [3], [11] montrent que ce matériau est constitué d'un assemblage de structures poreuses d'échelles bien différenciées. On distingue en général: - une "macroporosité " constituée de pores sphériques (d'un diamètre de l'ordre du millimètre) entourés d'une membrane solide;

- une porosité de la membrane solide provenant d'espaces (d'une taille d'environ $10 \mu \mathrm{m}$ ) séparant les agrégats de cristaux;

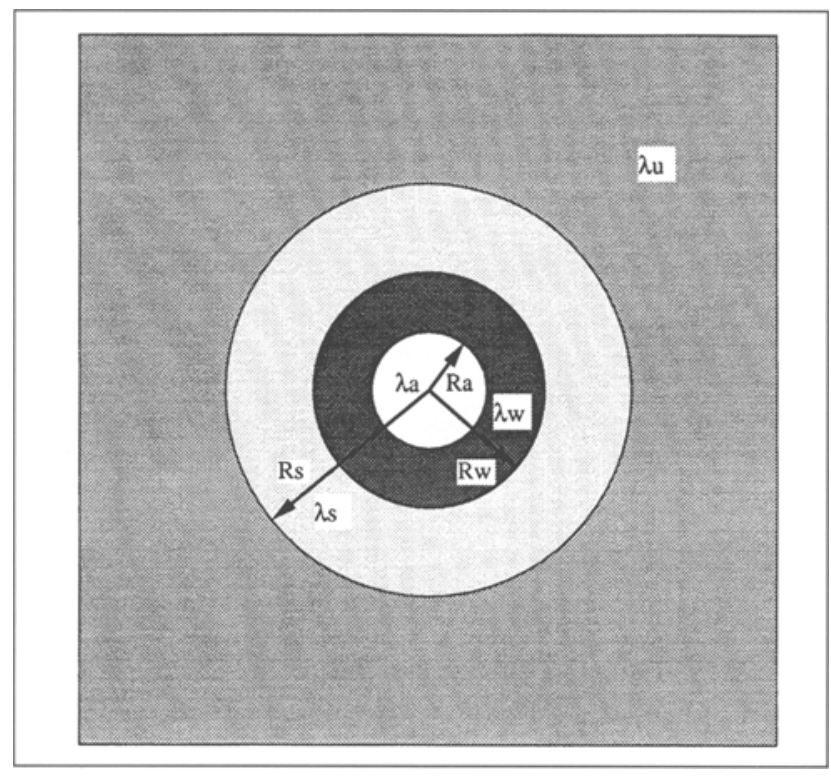

Fig. 3 - Géométrie d'une inclusion composite élémentaire prise en compte dans la méthode autocohérente appliquée aux milieux à trois phases.
- enfin, la porosité des agrégats cristallins $(\approx 0.1 \mu \mathrm{m})$.

Ces observations conduisent à penser que les hypothèses sur la microstructure faites dans l'approche autocohérente à inclusions composites, sont bien adaptées au cas $\mathrm{du}$ béton cellulaire. Notons cependant que l'adaptation n'est pas parfaite, car si des pores sphériques non connectés décrivent bien la macroporosité, ils ne donnent qu'une vision approchée de la porosité des agrégats cristallins.

\section{CONDUCTIVITÉ DU BÉTON CELLULAIRE SEC}

Appliquons maintenant ces résultats au béton cellulaire sec. Étant donné le mode de fabrication, on peut raisonnablement admettre que la masse volumique $\rho_{s}$ et la conductivité $\lambda_{s}$ des particules solides sont indépendantes de la masse volumique du béton cellulaire. En tenant compte de la faible conductivité de l'air par rapport à celle des particules solides $\left(\lambda_{\mathrm{a}} \ll<\lambda_{\mathrm{s}}\right)$ et exprimant la porosité totale $\varepsilon$ sous la forme :

$$
\rho_{\mathrm{d}}=\rho_{\mathrm{s}}(1-\varepsilon)
$$

on obtient à partir de l'expression (4)

$$
\begin{aligned}
\lambda_{\mathrm{d}} \approx \lambda_{\mathrm{s}}\left[1+\frac{\varepsilon}{\frac{1-\varepsilon}{3}-1}\right] & =\lambda_{\mathrm{s}} \frac{2}{3 /(1-\varepsilon)-1} \\
& =\lambda_{\mathrm{s}} \frac{2}{3 \rho_{\mathrm{s}} / \rho_{\mathrm{d}}-1}
\end{aligned}
$$

Cette formule n'est applicable que si l'on dispose d'une estimation de $\rho_{s}$ et $\lambda_{s}$. D'après les travaux [11], où des mesures fines par porosimétrie au mercure ont permis de donner la porosité totale d'échantillons de 340 à 720 $\mathrm{kg} / \mathrm{m}^{3}$, s peut être estimé à $2 \%$ près par : $\rho_{\mathrm{s}}=2650 \mathrm{~kg} / \mathrm{m}^{3}$.

Nous estimons $\lambda_{\mathrm{s}}$ à partir de la valeur usuelle de la conductivité $\lambda_{\mathrm{d}}=0.12 \mathrm{~W} / \mathrm{mK}$ du béton cellulaire de densité $\rho_{d}=500 \mathrm{~kg} / \mathrm{m}^{3}$. De l'expression (6) on déduit:

$$
\lambda_{\mathrm{s}}=\lambda_{\mathrm{d}}[3(2650 / 500)-1] / 2=0,894 \mathrm{~W} / \mathrm{mK}
$$

On notera que ces estimations de $s$ et $\lambda$ s correspondent aux valeurs usuelles pour des particules siliceuses ou calcaires.

Finalement la conductivité (en $\mathrm{W} / \mathrm{mK}$ ) s'exprime en fonction de la masse volumique $\left(\mathrm{en} \mathrm{kg} / \mathrm{m}^{3}\right.$ ) sous la forme :

$$
\lambda_{\mathrm{d}} \approx \frac{1,788}{\frac{7950}{\rho_{\mathrm{d}}}-1}
$$

Si l'on trace les variations de $\lambda_{\mathrm{d}}$ avec $\rho_{\mathrm{d}}$ données par (7), on observe que, dans la gamme des valeurs habituelles, la courbure est très faible, ce qui permet de remplacer la courbe par sa tangente au point $\rho_{\mathrm{d} 0}=500$ $\mathrm{kg} / \mathrm{m}^{3}$. En utilisant la formule de Taylor on est conduit à l'expression linéarisée suivante:

$$
\lambda_{\mathrm{d}}\left(\rho_{\mathrm{d}}\right) \approx \lambda_{\mathrm{d}}\left(\rho_{\mathrm{d} 0}\right)+\lambda_{\mathrm{d}}^{\prime}\left(\rho_{\mathrm{d} 0}\right) \cdot\left[\rho_{\mathrm{d}}-\rho_{\mathrm{d} 0}\right]
$$

$\operatorname{avec} \rho_{\mathrm{d} 0}=500 \mathrm{~kg} / \mathrm{m}^{3}$ 


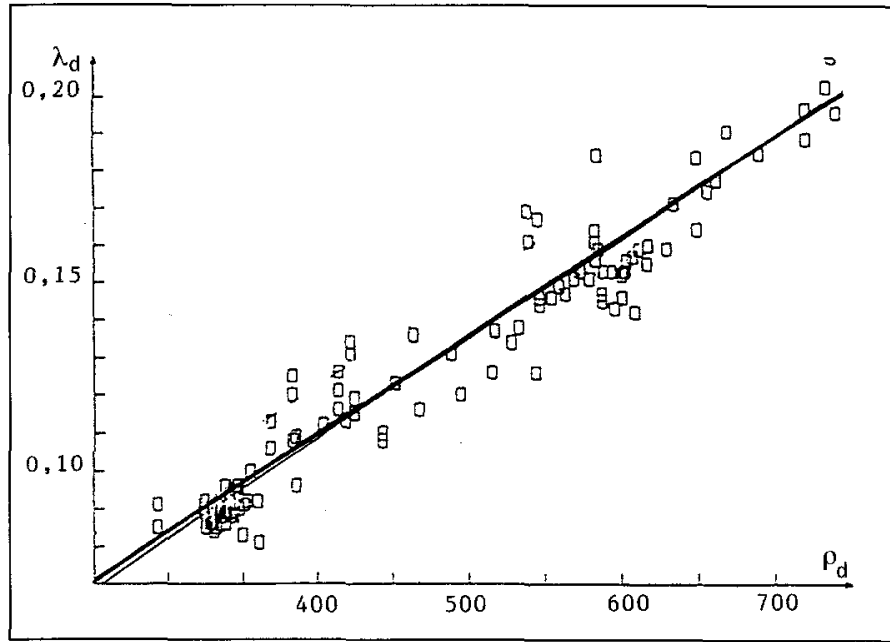

Fig. 4 - Conductivité à l'état sec, selon la valeur de la densité sèche. Comparaison entre calcul théorique selon l'expression (6) - trait gras -, formule empirique de Millard [4] - trait fin - et résultats expérimentaux - carrés -. Les résultats théoriques et empiriques sont pratiquement identiques, sauf très faibles densités.

soit :

$$
\lambda_{d}\left(\rho_{d}\right) \approx \frac{-2 \lambda_{s}}{\left(3 \rho_{s} / 500-1\right)^{2}}+\frac{6 \lambda_{s}}{\left(3 \rho_{s} / 500-1\right)^{2}} \cdot \frac{\rho_{s}}{500} \cdot \frac{\rho_{d}}{500}
$$

d'où, avec les paramètres déterminés précédemment :

$$
\lambda_{\mathrm{d}}\left(\rho_{\mathrm{d}}\right) \approx\left(-8,05+0,261 . \rho_{\mathrm{d}}\right) 10^{-3}
$$

La Fig. 4 montre le très bon accord entre ces expressions théoriques (6-8), des résultats expérimentaux, et la formule empirique proposée par Millard [4]:

$$
\lambda_{\mathrm{d}}\left(\rho_{\mathrm{d}}\right) \approx\left(-10,87+0,266 . \rho_{\mathrm{d}}\right) 10^{-3}
$$

\section{CONDUCTIVITÉ DU BÉTON CELLULAIRE HUMIDE}

Essayons d'appliquer maintenant la méthode autocohérente au béton cellulaire humide. Pour prendre en compte la présence de l'eau, deux approches différentes sont proposées.

\subsection{Modélisation par sphères composites à 3 phases}

Dans ce paragraphe nous supposerons que l'eau, de conductivité $\lambda_{\mathrm{w}}$, se répartit suivant une pellicule à la surface des pores (Fig. 3). La concentration d'eau dans les pores $\delta$ peut s'exprimer en fonction de la teneur en eau massique $u_{m}$ :

$$
\mathrm{u}_{\mathrm{m}}=\left(\delta . \rho_{\mathrm{w}}\right) /\left(\rho_{\mathrm{d}} / \varepsilon\right)
$$

soit :

$$
\delta=u_{m} \frac{\rho_{d}}{\rho_{w}\left(1-\rho_{d} / \rho_{s}\right)}
$$

Du fait de la faible conductivité de l'air $\left(\lambda_{\mathrm{a}}<<\lambda_{\mathrm{w}}, \lambda_{\mathrm{s}}\right)$, la conductivité du béton cellulaire humide $\lambda_{\mathrm{u}}$ s'exprime

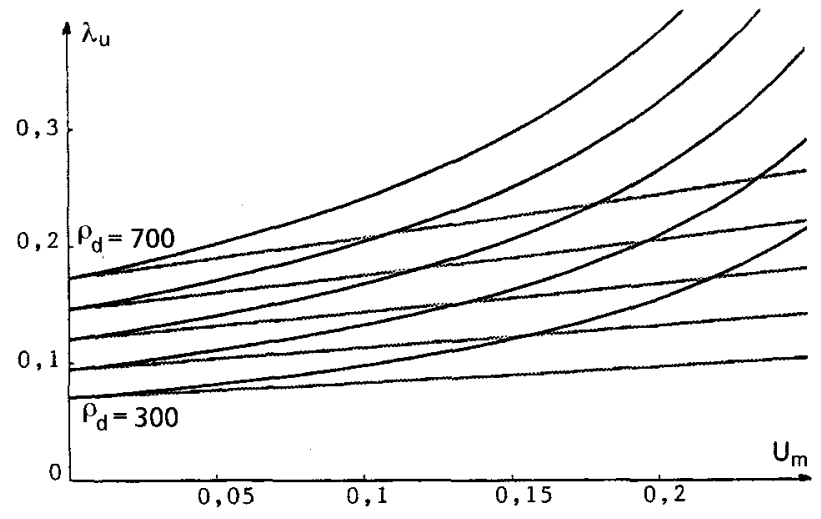

Fig. 5 - Calculs théoriques de la conductivité à l'état humide, selon la teneur en eau pour des densités sèches de $300,400,500$, $600,700 \mathrm{~kg} / \mathrm{m}^{3}$. Trait fin : calcul par inclusion triphasée (expression (9)). Trait gras : calcul par «double homogénéisation" (expression (13)).

sous la forme de (5):

$$
\lambda_{\mathrm{u}} \approx \lambda_{\mathrm{s}}\left\{1+\frac{\varepsilon}{\frac{1-\varepsilon}{3}-\frac{1-\delta / 3}{1-\delta\left(2 \lambda_{\mathrm{w}} / \lambda_{\mathrm{s}}+1\right) / 3}}\right\}
$$

avec $\varepsilon=1-\rho_{d} / \rho_{s}$

Ce résultat donnant la conductivité à l'état humide, selon la densité sèche et la teneur en eau, est représenté sous forme graphique en Fig. 5. On note la dépendance quasi linéaire de la conductivité avec la teneur en eau, ce qui justifie la linéarisation de l'expression par la formule de Taylor au voisinage de zéro:

$$
\lambda_{\mathrm{u}}\left(\mathrm{u}_{\mathrm{m}}\right) \approx \lambda_{\mathrm{u}}(0)+\lambda_{\mathrm{u}}^{\prime}(0) \cdot \mathrm{u}_{\mathrm{m}}=\lambda_{\mathrm{d}}+\lambda_{\mathrm{u}}^{\prime}(0) \cdot \mathrm{u}_{\mathrm{m}}
$$

soit :

$$
\lambda_{\mathrm{u}}\left(\mathrm{u}_{\mathrm{m}}\right) \approx \lambda_{\mathrm{d}}+\frac{\lambda_{\mathrm{w}}}{\left(1-\rho_{\mathrm{d}} / 3 \rho_{\mathrm{s}}\right)^{2}} \cdot \frac{2 \rho_{\mathrm{d}}}{3 \rho_{\mathrm{w}}} \cdot \mathrm{u}_{\mathrm{m}}
$$

ou encore, compte tenu de l'expression de $\lambda_{\mathrm{d}}$ :

$$
\left.\lambda_{\mathrm{u}}\left(\mathrm{u}_{\mathrm{m}}\right) \approx \lambda_{\mathrm{d}} \cdot \mid 1+\frac{1}{1-\rho_{\mathrm{d}} / 3 \rho_{\mathrm{s}}} \cdot \frac{\lambda_{\mathrm{w}}}{\lambda_{\mathrm{s}}} \cdot \frac{\rho_{\mathrm{s}}}{\rho_{\mathrm{w}}} \cdot \mathrm{u}_{\mathrm{m}}\right\rceil
$$

Sous ces formes linéarisées (7) et (8), il est facile de comparer nos résultats à ceux de la littérature $[4,5]$. Par exemple, pour $\rho_{\mathrm{d}}=500 \mathrm{~kg} / \mathrm{m}^{3}$, on trouve :

$$
\lambda_{\mathrm{u}}\left(\mathrm{u}_{\mathrm{m}}\right) \approx \lambda_{\mathrm{d} \cdot} \cdot\left[1+1,91 \cdot \mathrm{u}_{\mathrm{m}}\right]
$$

alors que selon les normes :

$$
\lambda_{\mathrm{u}}\left(\mathrm{u}_{\mathrm{m}}\right) \approx \lambda_{\mathrm{d}} \cdot\left[1+4,00 \cdot \mathrm{u}_{\mathrm{m}}\right]
$$

Il apparait donc que l'approche autocohérente sousestime l'influence de la teneur en eau. Cette conclusion est confirmée en Fig. 6 où l'on présente une comparaison entre les valeurs théoriques et expérimentales de la pente des courbes $\left(\lambda_{\mathrm{u}}, \mathrm{u}_{\mathrm{m}}\right)$ en fonction de la densité sèche. On notera cependant que, sous l'aspect qualitatif, 


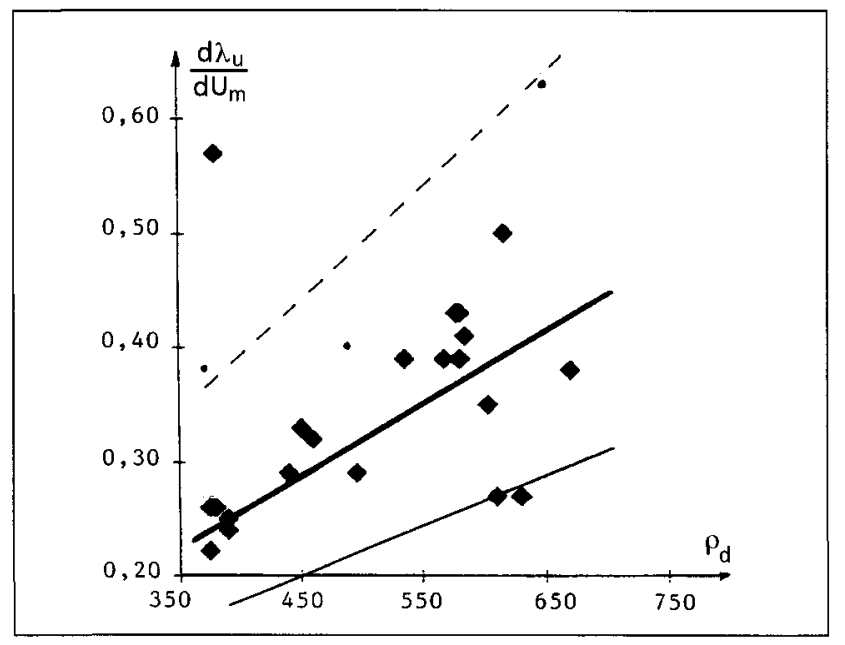

Fig. 6 - Comparaison entre les valeurs théoriques et expérimentales de la pente des courbes $\left(\lambda_{u}, u_{m}\right)$ en fonction de la densité sèche. Losanges (d'après [5]), points (d'après [4]) : valeurs expérimentales. Trait fin : calcul par inclusion triphasée (expression (10)). Trait gras : calcul par "double homogénéisation» (expression (15)). Trait pointillé : pente donnée par la norme $\left(4 . \lambda_{\mathrm{d}}\right)$.

les variations avec $\rho_{\mathrm{d}}$ de la pente théorique donnée par l'expression (10):

$$
\frac{\lambda_{\mathrm{w}}}{\left(1-\rho_{\mathrm{d}} / 3 \rho_{\mathrm{s}}\right)^{2}} \cdot \frac{2 \rho_{\mathrm{d}}}{3 \rho_{\mathrm{w}}}
$$

sont comparables à celles obtenues expérimentalement.

Il est probable que cet accord qualitatif mais non quantitatif provienne de l'hypothèse faite pour décrire le béton cellulaire humide. En effet, celle-ci consiste à supposer que la concentration d'eau dans les pores est indépendante de leur taille, alors que dans la réalité elle est d'autant plus forte que les pores sont petits.

Dans le paragraphe suivant nous proposons une description plus conforme à la répartition réelle de l'eau dans les pores.

\subsection{Modélisation par «double» homogénéisation}

Pour mieux traduire la répartition de l'eau qui occupe essentiellement les pores de petite taille, nous considérons que les bulles d'air sont entourées par un

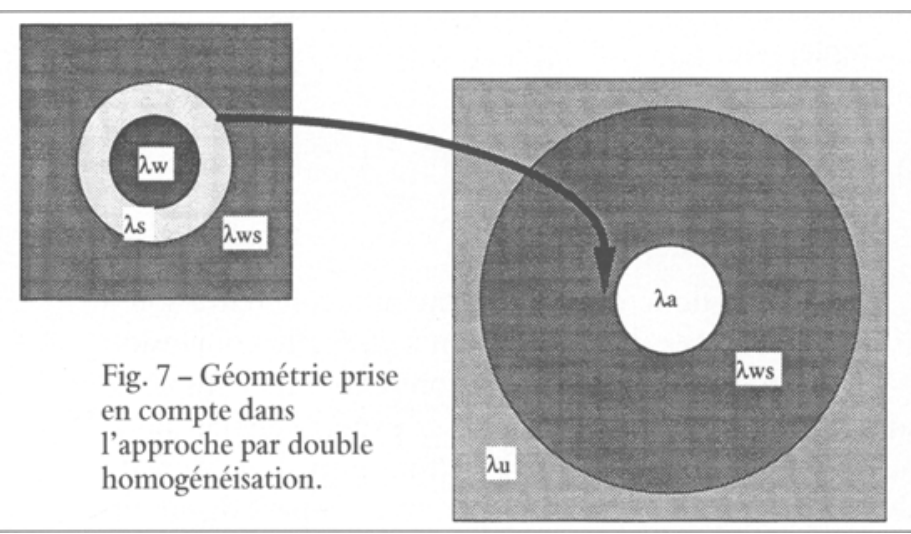

milieu poreux saturé d'eau (Fig. 7). Nous procéderons donc par double homogénéisation, en déterminant dans un premier temps la conductivité équivalente du milieu saturé puis la conductivité d'ensemble. Cette approche suppose implicitement les pores saturés beaucoup plus petits que les pores secs, pour qu'à l'échelle de ces derniers la membrane saturée puisse être considérée comme un milieu homogène.

\section{Conductivité du milieu saturé}

Suivant cette description, la masse d'un volume donné de béton cellulaire humide est celle du milieu saturé. La concentration volumique d'eau $\varepsilon_{\mathrm{w}}$ dans ce milieu est donc directement reliée à la teneur en eau massique de $\mathrm{AAC}$ par la relation:

$$
\varepsilon_{\mathrm{w}}=\mathrm{u}_{\mathrm{m}} \frac{\rho_{\mathrm{s}}}{\rho_{\mathrm{w}}}
$$

En appliquant les résultats du paragraphe 2.1, la conductivité du milieu saturé est :

$$
\lambda_{\mathrm{ws}}=\lambda_{\mathrm{s}} \cdot\left[1+\frac{\varepsilon_{\mathrm{w}}}{\frac{1-\varepsilon_{\mathrm{w}}}{3}-\mathrm{r}}\right]
$$

avec:

$$
r=\frac{1}{1-\lambda_{w} / \lambda_{s}}
$$

\section{Conductivité d'ensemble}

Avec des bulles à la concentration $\varepsilon_{a}$, la conductivité d'ensemble s'obtient à nouveau par la formule (5):

$$
\lambda_{\mathrm{u}}=\lambda_{\mathrm{ws}} \cdot\left[1+\frac{\varepsilon_{\mathrm{a}}}{\frac{1-\varepsilon_{\mathrm{a}}}{3}-1}\right] \quad\left(\text { puisque } \lambda_{\mathrm{a}} \approx 0\right. \text { ) }
$$

soit

$$
\lambda_{\mathrm{u}}=\lambda_{\mathrm{s}} \cdot\left[1+\frac{\varepsilon_{\mathrm{a}}}{\frac{1-\varepsilon_{\mathrm{a}}}{3}-1}\right] \cdot\left[1+\frac{\varepsilon_{\mathrm{w}}}{\frac{1-\varepsilon_{\mathrm{w}}}{3}-\mathrm{r}}\right]
$$

Pour que cette description par double homogénéisation traduise effectivement les propriétés du matériau, considérons le cas où le béton cellulaire est à l'état sec. La première étape donnerait alors une conductivité égale à :

$$
\lambda_{\mathrm{s}} \cdot\left[1+\frac{\varepsilon_{\mathrm{w}}}{\frac{1-\varepsilon_{\mathrm{w}}}{3}-\mathrm{r}}\right]
$$

et la deuxième étape conduirait à :

$$
\lambda_{\mathrm{u}}(\mathrm{u}=0)=\lambda_{\mathrm{d}}=\lambda_{\mathrm{s}} \cdot\left[1+\frac{\varepsilon_{\mathrm{a}}}{\frac{1-\varepsilon_{\mathrm{a}}}{3}-1}\right] \cdot\left[1+\frac{\varepsilon_{\mathrm{w}}}{\frac{1-\varepsilon_{\mathrm{w}}}{3}-1}\right]
$$


d'où en comparant avec (6):

$$
\left[1+\frac{\varepsilon}{\frac{1-\varepsilon}{3}-1}\right]=\left[1+\frac{\varepsilon_{\mathrm{a}}}{\frac{1-\varepsilon_{\mathrm{a}}}{3}-1}\right] \cdot\left[1+\frac{\varepsilon_{\mathrm{w}}}{\frac{1-\varepsilon_{\mathrm{w}}}{3}-1}\right]
$$

on en déduit que :

$$
\lambda_{\mathrm{u}}=\lambda_{\mathrm{d}} \cdot\left[1+\frac{\varepsilon_{\mathrm{w}}}{\frac{1-\varepsilon_{\mathrm{w}}}{3}-\mathrm{r}}\right] \cdot\left[1+\frac{\varepsilon_{\mathrm{w}}}{\frac{1-\varepsilon_{\mathrm{w}}}{3}-1}\right]^{2}
$$

Soit sous une forme plus simple:

$$
\lambda_{\mathrm{u}}=\lambda_{\mathrm{d}} \cdot\left[1+\frac{9(\mathrm{r}-1)}{2} \frac{\varepsilon_{\mathrm{w}}}{\left(1-\varepsilon_{\mathrm{w}}\right)\left(3 \mathrm{r}-1+\varepsilon_{\mathrm{w}}\right)}\right]
$$

avec:

$$
\varepsilon_{\mathrm{w}}=\mathrm{u}_{\mathrm{m}} \frac{\rho_{\mathrm{s}}}{\rho_{\mathrm{w}}}
$$

On présente en Fig. 5 une illustration de ce résultat. La comparaison avec l'approche précédente montre une dépendance plus forte et "moins linéaire" de la conductivité avec la teneur en eau.

Pour les teneurs en eau inférieures à 20\%, en assimilant les courbes à leur tangente à l'origine, et en tenant compte de l'expression de $\mathrm{r}$, on obtient l'approximation suivante:

$$
\lambda_{\mathrm{u}} \approx \lambda_{\mathrm{d}} \cdot\left[1+\frac{9}{2\left(2+\lambda_{\mathrm{w}} / \lambda_{\mathrm{s}}\right)} \frac{\lambda_{\mathrm{w}} \rho_{\mathrm{s}}}{\lambda_{\mathrm{s}} \rho_{\mathrm{w}}} \mathrm{u}_{\mathrm{m}}\right]
$$

ce qui donne numériquement : $\lambda_{\mathrm{u}} \approx \lambda_{\mathrm{d} \cdot[}\left[1+3,45 \cdot \mathrm{u}_{\mathrm{m}}\right]$

Cette relation est en bon accord avec celle fixée par les normes, (13), d'autant plus que le coefficient 3,45, obtenu par la pente à l'origine, donne une sous-estimation de la dépendance avec $\mathrm{u}_{\mathrm{m}}$.

Ces résultats théoriques sont confrontés aux mesures expérimentales en Fig. 6. Par rapport à la première modélisation, la valeur théorique de la pente des courbes $\left(\lambda_{u}, u_{m}\right)$ en fonction de la densité sèche, donnée par:

$$
\frac{9 \lambda_{w}}{2+\lambda_{w} / \lambda_{s}} \frac{\rho_{s}}{\rho_{w}} \frac{1}{3 \rho_{s} / \rho_{d}-1}
$$

est en meilleur accord avec les expériences.

Ainsi, pour les teneurs en eau usuelles, l'approche par "double homogénéisation" semble mieux traduire la microstructure et donne une description satisfaisante des propriétés thermiques du béton cellulaire humide.

\section{CONCLUSION}

L'application de la méthode autocohérente à inclusions composites permet de caractériser simplement la conductivité thermique du béton cellulaire.

Dans le cas où le matériau est sec, les résultats (équations 6,7$)$ sont en parfait accord avec les mesures expéri- mentales. On propose ainsi une justification théorique des formules empiriques connues.

Lorsque le matériau est humide, les résultats obtenus avec des inclusions composites triphasées (équation 11) sont seulement en accord qualitatif avec l'expérience. Ceci provient d'une description trop sommaire de la répartition de l'eau dans les pores.

Par contre, l'approche par "double homogénéisation", qui traduit mieux la microstructure du béton cellulaire humide, donne des estimations satisfaisantes (équation 14). La différence entre ces deux descriptions met clairement en évidence le rôle essentiel de la répartition de l'eau.

En conclusion, malgré les hypothèses simplificatrices du modèle autocohérent, on obtient une description acceptable, de la conductivité du béton cellulaire. L'avantage de cette méthode est, d'une part de donner expressions analytiques simples, et d'autre part, de mettre clairement en évidence les paramètres essentiels qui déterminent la conductivité d'ensemble du matériau.

\section{REMERCIEMENTS}

Je remercie M. Guegan (SIPOREX) pour le financement de cette étude.

\section{RÉFÉRENCES}

[1] Hums, D., 'Relation between humidity and heat conductivity in aerated concrete' in 'Autoclaved Aerated Concrete, Moisture and Properties. Developments in Civil Engineering', 6 (1983), Ed Wittmann, F.H. (Elsevier) 143-152.

[2] Loudon, A. G., 'The effect of moisture content on thermal conductivity' in 'Autoclaved Aerated Concrete, Moisture and Properties. Developments in Civil Engineering' 6 (1983), Ed. Wittmann, F.H. (Elsevier) 131-142.

[3] Tada, S. et Nakano, S., 'Microstructural approach to properties of moist cellular concrete', in 'Autoclaved Aerated Concrete, Moisture and Properties. Developments in Civil Engineering', 6 (1983), Ed. Wittmann, F.H. (Elsevier) 71-91.

[4] Millard, W.R., 'The thermal performance of European aerated concrete' in 'Advances in Autoclaved Aerated Concrete', Ed. Wittmann, F.H. (Balkema, 1992), 83-88.

[5] Frey, E., 'Recent results on thermal conductivity and hygroscopic moisture content of AAC', in 'Advances in Autoclaved Aerated Concrete', Ed. Wittmann, F.H. (Balkema, 1992), 89-92.

[6] Laurent, J. P. et Frendo-Rosso, C., 'Application of image analysis to the estimation of AAC thermal conductivity', in 'Advances in Autoclaved Aerated Concrete', Ed. Wittmann, F.H. (Balkema, 1992), 89-92.

[7] Kröner, E., 'Berechnung der elastischen konstanten des vielkristalls aus den konstanten des einkristalls', Z. Phys. 151 (1958) 504.

[8] Hashin, Z., 'Assessment of the self consistent scheme approximation: Conductivity of particulate composites', J. Comp. Mater. 2 (1968) 284.

[9] Herve, E. et Zaoui, A., 'Modelling the effective behavior of non linear matrix-inclusion composites', Eur. J. Mech. A/Solids, 9 (6) (1990) 505-515.

[10] Kröner, E. "Self-consistent scheme and graded disorder in polycrystal elasticity', J. Phys. F., 8 (1978), 2261.

[11] Prim, P. et Wittmann, F. H. 'Structure and water absorption of aerated concrete', in 'Autoclaved Aerated Concrete, Moisture and Properties. Developments in Civil Engineering', 6, Ed. Wittmann, F.H. (Elsevier, 1983) 55-70. 